\title{
Correction to: Simulated interannual variation in summertime atmospheric circulation associated with the East Asian monsoon
}

\author{
Miki Arai $^{1} \cdot$ Masahide Kimoto ${ }^{1}$
}

Published online: 22 June 2018

๑) Springer-Verlag GmbH Germany, part of Springer Nature 2018

\section{Correction to: Clim Dyn (2008) 31:435-447 https://doi.org/10.1007/s00382-007-0317-y}

We found an unintentional error in the calculation of monthly anomalies used in our original article. As the monthly anomalies were used for depicting probability density function projected onto EOF1 and EOF2 of JJA-averaged anomalies (Fig. 9), we redraw the probability density function and the composite difference map between cold and hot summer regime (Fig. 10) defined by two peaks in Fig. 9 in the previous paper.

After the replacement of newly defined monthly anomalies to calculation for depicting the probability density function, the two peaks can still be recognized although the bimodality is not so distinct compared to those in Fig. 9 of the previous paper. Moreover, the feedback from Rossby waves with sub-monthly time scale in cold summer regime as shown in Fig. 10 in the previous paper can be obviously seen.

We concluded that the results with newly defined monthly anomaly dataset did not change our previous suggestion.

In our previous paper (hereafter, AK08), we found an unintentional error in the calculation of monthly anomalies used to plot the probability density function (PDF) in the original Fig. 9. The monthly anomalies were calculated in AK08 as the deviation from the JJA seasonal mean climatology rather than the monthly climatology, as originally intended. Therefore, the anomalies used for Fig. 9 included the mean seasonal cycle component, although we intended to investigate the interannual variation. The summertime

The original article can be found online at https://doi.org/10.1007/ s00382-007-0317-y.

Miki Arai

arai@aori.u-tokyo.ac.jp

1 Atmosphere and Ocean Research Institute, University of Tokyo, 5-1-5, Kashiwanoha, Kashiwa, Chiba 277-8568, Japan atmospheric circulation over East Asia changes from early summer to midsummer and is accompanied by the northward advance of the Baiu front (Ninomiya and Murakami 1987). Therefore, the distinct bimodality shown in Fig. 9 of AK08 was dominated by the average seasonal migration from early summer (cold summer regime) to midsummer (hot summer regime).

To investigate the interannual variation in atmospheric circulation over the East Asian monsoon area as originally intended, we defined a renewed monthly anomaly field for each month as the difference from the ensemble averaged monthly climatology for 20 years. The observational and simulated data sets used here are the same as those used in AK08.

Figure 1a shows a PDF of the renewed monthly anomaly field of the PD experiment (PD_high in AK08) projected onto EOF1 and EOF2, which were obtained from JJA-averaged anomalies and were the same as those used in the original Fig. 9a. We recognized two regimes in the renewed PDF, although the bimodality was not as distinct as in Fig. 9a in AK08 and the center of both regimes approached the origin. The projected distribution in closer to the origin since monthly anomaly data projected more onto higher EOF modes. The two regimes were located in the positive and negative projection onto PC1. The two peaks in the PC phase space were also shown in the renewed PDF of the observation (Fig. 1c), although they were less obvious than those of the simulation because of the smaller sample size. However, the trace of bimodality can still be recognized in the renewed figure.

A PDF of the doubled $\mathrm{CO}_{2}$ experiment $\left(2 \mathrm{CO}_{2}\right)$ corresponding to Fig. $9 \mathrm{~b}$ in AK08 is shown in Fig. 1b. Two regimes were recognized as those in the PD experiment; the residence frequency of the cold summer regime was increased, whereas that of the hot summer regime was decreased compared to the PD experiment (Fig. 1a). This suggests that a climate regime shift due to global warming 

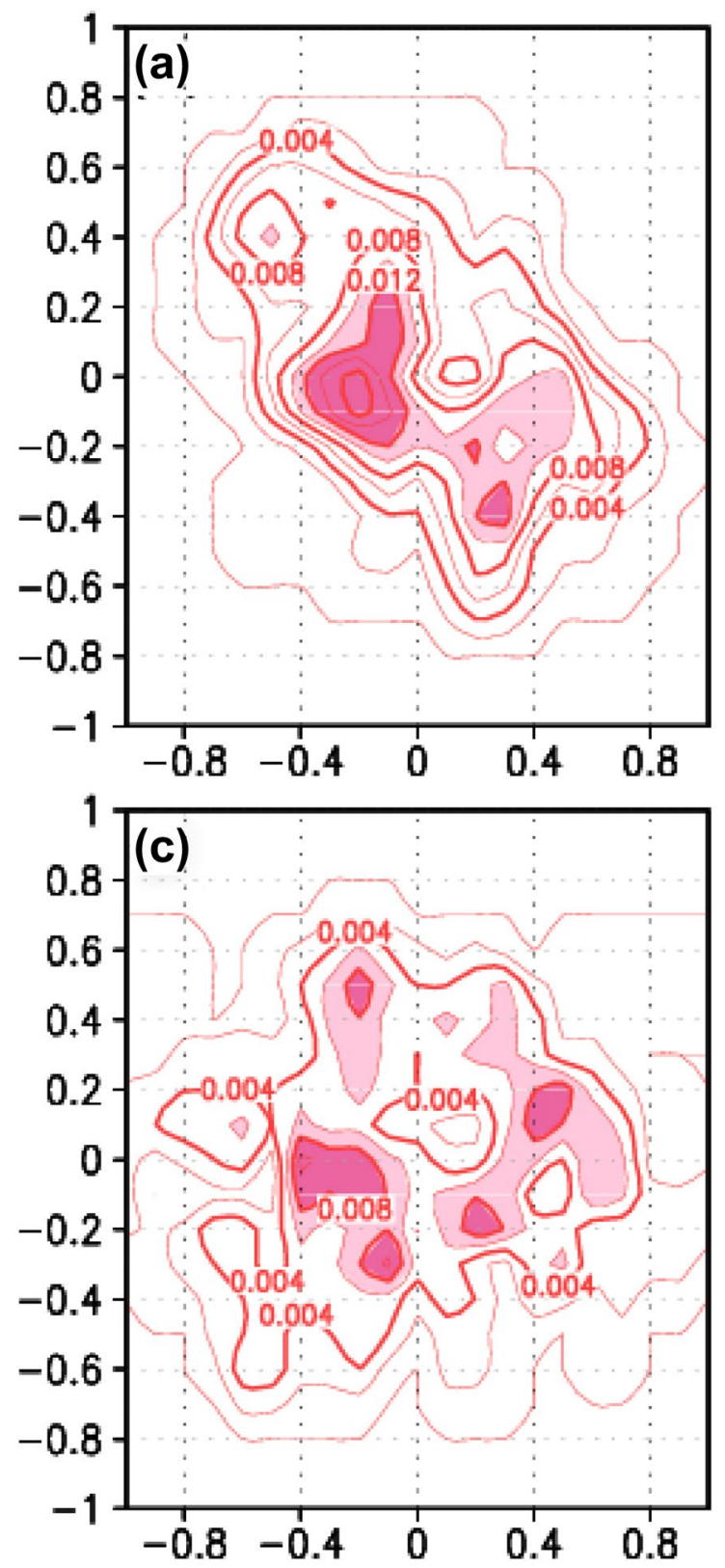

Fig. 1 PDF constructed by a renewed monthly anomaly field over the summertime East Asian monsoon area for $\mathbf{a}$ the PD experiment, $\mathbf{b}$ the $2 \mathrm{CO}_{2}$ experiment, and $\mathbf{c}$ observation (ECMWF ERA40). The basis is $\mathrm{PC} 1$ and PC2 of the EOF analysis for the ensemble averaged anomaly

(Palmer 1999; Corti et al. 1999) existed in the simulated summertime East Asian circulation as shown in AK08.

Because the definitions of cold and hot summer regimes used to depict Fig. 10 in AK08 were based on the two regimes seen in Fig. 9, we redrew Fig. 10 using the renewed monthly anomaly field. We defined the mean flow and the transient part as the composites of the newly defined cold/hot summer regime from Fig. 1 and 11-day running averaged daily data, respectively, as in the original paper. The difference between the composites of the

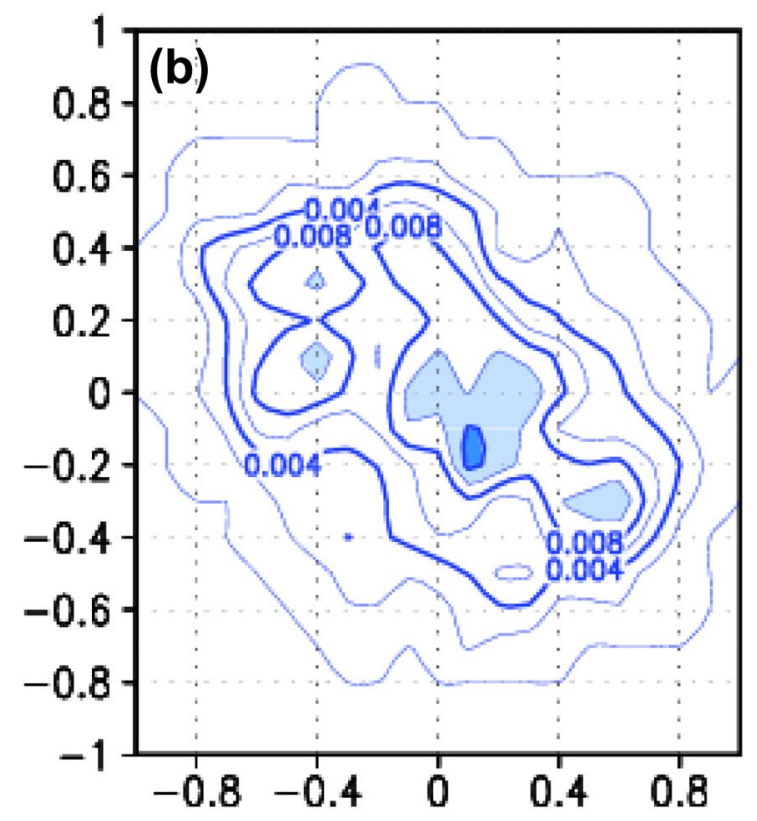

field of geopotential height on the $500 \mathrm{hPa}$ level. These figures are the counterparts of Fig. 9 in AK08. The contour intervals are 0.0025 in all panels. Light shading denotes an exceedance of 0.01 , whereas heavy shading denotes an exceedance of 0.0175

cold/hot summer regime of the wave activity (Takaya and Nakamura 2001), meridional component of potential vorticity flux, and potential vorticity at the $250 \mathrm{hPa}$ level of observation is shown in Fig. $2 \mathrm{a}$ and (for the PD experiment) Fig. 2b, which corresponds to Fig. 10 in AK08. The members used to make composites were only 8 (cold summer regime) and 9 (hot summer regime) for the observations. A diffluent tendency of mean flow in the cold summer regime was seen over eastern Siberia $\left(100^{\circ} \mathrm{E}-120^{\circ} \mathrm{E}\right)$, and a remarkable convergence area of the transient PV 

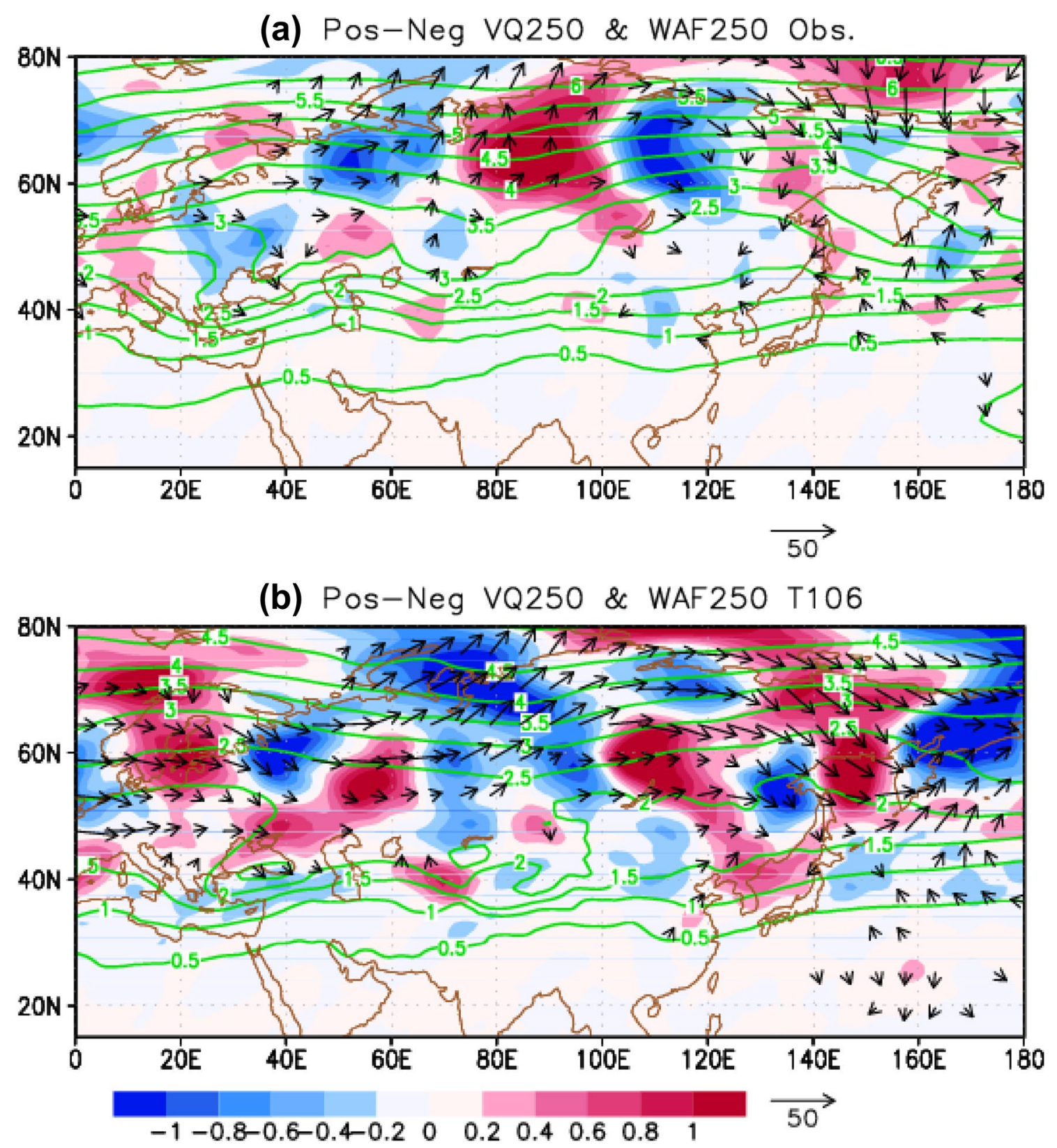

Fig. 2 The difference in wave activity flux (arrow) and meridional component of potential vorticity flux (shade, unit: $10^{-6} \mathrm{~m}^{2} \mathrm{~s}^{-2} \mathrm{~kg}^{-1} \mathrm{~K}$ ) at the $250 \mathrm{hPa}$ level between the cold and hot

flux (negative shading) was located in this area. The wave activity flux also converged over $100^{\circ} \mathrm{E}-120^{\circ} \mathrm{E}$. The composite difference for the PD experiment, consisting of 68 months for the cold summer regime and 51 months for the hot summer regime, showed more significant feedback from the transient eddy to the mean flow than that shown for the observation. The eastward propagation of wave activity flux for the cold summer regime was emphasized, and convergence can be seen over eastern Siberia $\left(120^{\circ} \mathrm{E}-140^{\circ} \mathrm{E}, 50^{\circ} \mathrm{N}-70^{\circ} \mathrm{N}\right)$ in correspondence with a summer regimes a with observation and $\mathbf{b}$ with PD. The climatology of the ensemble averaged potential vorticity in JJA is also depicted by green solid lines (unit: $10^{-6} \mathrm{~m}^{2} \mathrm{~s}^{-1} \mathrm{~kg}^{-1} \mathrm{~K}$ )

convergence of the transient PV flux (Fig. 2b). These features of the composite difference are the same as those in Fig. 10 in AK08.

The reexamination with a renewed monthly mean anomaly field showed that the feedback from Rossby waves with a submonthly timescale to a diffluent mean flow with a monthly to seasonal timescale still works. We again suggest that the eddy feedback process illustrated in Fig. 11 in AK08 contributes to the bimodality of interannual variation in summertime East Asian circulation. 
Acknowledgements This study was supported by the Core Research for Environmental Science and Technology of Japan Science and Technology Agency, and by the Kyosei and Kakushin project of ministry of education, culture, sports, science, and technology, and by Global Environmental Research Fund (GERF) of Ministry of Environment of Japan.

\section{References}

Corti S, Molteni F, Palmer TN (1999) Signature of recent climate change in frequencies of natural atmospheric circulation regimes. Nature 398:799-801
Ninomiya K, Murakami T (1987) The early summer rainy season (Baiu) over Japan. In: Chang C-P, Krishnamurti TN (eds) Monsoon meteorology. Oxford University Press, Oxford, pp 93-121

Palmer TN (1999) A nonlinear dynamical perspective on climate prediction. J Clim 12:575-591

Takaya K, Nakamura H (2001) A Formulation of a phase-independent wave-activity flux for stationary and migratory quasigeostrophic eddies on a zonally varying basic flow. J Atmos Sci 58(6):608-627 